\title{
Composição corporal de estudantes adultos com deficiência intelectual e síndrome de Down
}

\author{
Body composition of adult students with intellectual disability and Down \\ syndrome
}

Composición corporal de estudiantes adultos con discapacidad intelectual y síndrome de Down

\section{Lívia Caroline Nunes do Nascimento}

Graduada pelo Centro Universitário São Lucas, Porto Velho, Rondônia, Brasil. liviacnn@hotmail.com

ORCID - https://orcid.org/0000-0001-8430-8667

\section{Luna Mares Lopes de Oliveira}

Professora doutora no Centro Universitário São Lucas, Porto Velho, Rondônia, Brasil. lunamares@saolucas.edu.br ORCID - https://orcid.org/0000-0002-4756-460X

Danielle Souza Nogueira

Graduada pelo Centro Universitário São Lucas, Ji-Paraná, Rondônia, Brasil. daniellesng@hotmail.com ORCID - https://orcid.org/0000-0003-1911-5054

\section{Elane Regina Andrade}

Graduada pelo Centro Universitário São Lucas, Porto Velho, Rondônia, Brasil.

elane_regina_pvh@hotmail.com

ORCID - https://orcid.org/0000-0002-0221-9204

\section{Layane Ferreira Feitoza}

Graduada pelo Centro Universitário São Lucas, Ji-Paraná, Rondônia, Brasil. layane_pvh@hotmail.com

ORCID - https://orcid.org/0000-0002-0224-2738

Recebido em 27 de agosto de 2019

Aprovado em 11 de março de 2020

Publicado em 9 de abril de 2020

\section{RESUMO}

A deficiência intelectual (DI) é caracterizada por limitações significativas em funcionamento intelectual e em habilidades adaptativas. A síndrome de Down (SD) é uma condição humana geneticamente determinada com características próprias. O objetivo deste estudo foi avaliar a composição corporal associada ao risco de surgimento de comorbidades cardiovasculares e metabólicas em estudantes adultos com DI leve e moderada e SD, em uma instituição pública do município de Porto Velho-RO. Trata-se de um estudo do tipo transversal, com análise quantitativa dos dados, no qual foram coletados peso e altura dos participantes para posterior cálculo do Índice de Massa Corporal (IMC), bem como a verificação das circunferências do braço, cintura, quadril e dobras cutâneas (subescapular, supra ilíaca, bicipital e tricipital) para a análise do 
percentual de gordura corporal. Pesquisa realizada com 34 alunos, sendo 21 do gênero masculino e 13 do feminino. Destes, 85\% diagnosticados com DI e 15\% com SD. Verificou-se que os alunos com DI eram mais altos que os SD, no entanto estes últimos apresentaram IMC mais elevado. Quanto ao risco de doenças cardiovasculares e metabólicas, pelas medidas de circunferência da cintura e relação cintura-quadril, os alunos com SD foram classificados com risco muito elevado. A massa muscular avaliada pela circunferência muscular do braço $(\mathrm{CMB})$ apresentou maior prevalência de ausência de risco, embora uma parcela de alunos com DI tenha sido classificada com desnutrição leve por este indicador. Tanto a desnutrição quanto o excesso de peso devem ser levados em consideração nas políticas de saúde para alunos especiais.

Palavras-chave: Estado nutricional; Síndrome de Down; deficiência intelectual.

\section{ABSTRACT}

Intellectual disability (ID) is characterized by significant limitations in intellectual functioning and adaptive skills. Down syndrome (DS) is a genetically determined human condition with its own characteristics. The aim of this study was to evaluate the body composition associated with the risk of cardiovascular and metabolic comorbidities in adult students with mild and moderate ID and DS in a public institution in Porto Velho-RO. This is a cross-sectional study, with quantitative data analysis, in which participants' weight and height were collected for subsequent calculation of Body Mass Index (BMI), as well as the verification of arm circumference, the waist and hip, skinfolds (subscapular, supra iliac, bicipital and tricipital) for the analysis of body fat percentage. The study was conducted with 34 students, 21 males and 13 females, among which $85 \%$ were diagnosed with ID and $15 \%$ with DS. Results indicated that students with ID were taller than students with DS, however they presented higher BMI. Regarding the risk of cardiovascular and metabolic diseases by measures of waist circumference and waist-hip ratio, students with DS were classified at very high risk. Muscle mass assessed by mid-arm muscle circumference (MAMC) had a higher prevalence of non-risk, although a part of students with ID was classified as mildly malnourished by this indicator. Both, malnutrition and overweight, should be taken into account in special students' health policies.

Keywords: Nutritional status; Down's Syndrome; intellectual disability.

\section{RESUMEN}

La discapacidad intelectual (DI) se caracteriza por limitaciones significativas en el funcionamiento intelectual y las habilidades de adaptación. El síndrome de Down (SD) es una condición humana genéticamente determinada con sus propias características. El objetivo de este estudio fue evaluar la composición corporal asociada con el riesgo de comorbilidades cardiovasculares y metabólicas en estudiantes adultos con DI y SD leve y moderada en una institución pública en Porto Velho-RO. Este es un estudio transversal, con análisis de datos cuantitativos, en que se recolectó el peso y la altura de los participantes para el cálculo posterior del índice de masa corporal (IMC), así como la verificación de la circunferencia del brazo, cintura, cadera y pliegues cutáneos (subescapular, supra ilíaco, bicipital y tríceps) para el análisis del porcentaje de grasa corporal. La investigación se realizó con 34 estudiantes, 21 hombres y 13 mujeres. De estos, $85 \%$ diagnosticados con DI y $15 \%$ con SD. Se encontró que los estudiantes con ID eran más altos que los estudiantes con SD, sin embargo, este último presentó un IMC más alto. Con respecto al riesgo de enfermedades cardiovasculares y metabólicas por medidas de circunferencia de la cintura y relación cintura-cadera, los estudiantes SD fueron clasificados como de muy alto riesgo. La masa muscular evaluada por la 
circunferencia muscular del brazo $(\mathrm{CMB})$ presentó una mayor prevalencia de no riesgo, aunque una porción de los estudiantes con ID fueron clasificadas como levemente desnutridos por este indicador. Tanto la desnutrición como el sobrepeso deben tenerse en cuenta en las políticas especiales de salud estudiantil.

Palabras clave: Estado nutricional; Sindrome de Down; discapacidad intelectual.

\section{Introdução}

No Censo brasileiro de 2010, 24\% dos entrevistados relataram possuir algum grau de dificuldade, sendo 1,4\% com deficiência mental/intelectual (IBGE, 2010). Pessoas com deficiência intelectual (DI) possuem maior vulnerabilidade nutricional devido às alterações metabólicas e fisiopatológicas que as predispõem a doenças crônicas não transmissíveis, como obesidade, doenças cardiovasculares, diabetes, hipertensão arterial, dislipidemia e síndrome metabólica, além de apresentarem, no consumo alimentar, alterações que afetam a ingestão de energia e nutrientes (BAGNI; OLIVEIRA; PINTO; SANTOS; ARAÚJO, 2015). Estudos realizados em vários países demonstraram que o uso de medicamentos antidepressivos, antipsicóticos e antiepiléticos e baixos níveis de atividade física no grupo de pessoas com deficiência são fatores que elevam o risco para a obesidade (WINTER et al., 2012).

O termo deficiência intelectual substituiu o termo retardo mental na 11 â edição do Definition Manual of the American Association on Intellectual and Developmental Disabilities (AAIDD, 2011). Essa condição é, na atualidade, definida como "uma incapacidade caracterizada por importantes limitações, tanto no funcionamento intelectual quanto no comportamento adaptativo", sendo diagnosticada antes dos 18 anos de idade. Vale destacar que as habilidades intelectuais incluem raciocínio, planejamento, resolução de problemas, pensamento abstrato, compreensão de ideias complexas e rapidez na aprendizagem (AAMR, 2006).

A DI é a deficiência mais comum de desenvolvimento, caracterizada por funcionamento intelectual significativamente abaixo da média, juntamente com limitações relacionadas em áreas como comunicação, autodireção, segurança, saúde e lazer ou do trabalho e das habilidades sociais (MAHAN; STUM; RAYMOND, 2012).

A síndrome de Down (SD) é um distúrbio genético e metabólico no qual ocorre uma alteração cromossômica, que pode ser de três tipos: trissomia 21 (presença e expressão de três cópias de genes localizados no cromossomo 21); translocação (um material 
http://dx.doi.org/10.5902/1984686X35273

cromossômico extra fica aderido a outro cromossomo e o paciente tem 46 cromossomos); e mosaicismo (podem originar-se da não disjunção mitótica nas primeiras divisões de um zigoto normal). A alteração genética compromete apenas parte das células, ou seja, algumas células têm 47 e outras 46 cromossomos (ocorre em cerca de $2 \%$ dos casos de síndrome de Down) (SILVA-JUNIOR; TORNELLO; GORLA; CALEGARI, 2007).

Gonçalves (2014) relata que pessoas com SD possuem características físicas comuns, como baixa estatura, nariz pequeno e achatado, hipotonia muscular, maior volume de pele no pescoço, taxa metabólica basal reduzida e consequente diminuição do gasto energético, que influencia diretamente no estado nutricional. Outra característica física comum em portadores de SD é o excesso de peso e a obesidade (SILVA; SANTOS; MARTINS, 2006). A obesidade, nesse grupo, normalmente está relacionada com alterações associadas aos aspectos genéticos, fisiológicos e ambientais (BERTAPELLI; GORLA; COSTA; FREIRE, 2011). Segundo dados encontrados em estudo feito com 104 adultos portadores de SD, $54,3 \%$ dos indivíduos foram classificados como obesos, sendo $62,7 \%$ das mulheres e $46,3 \%$ dos homens (SILVA; SANTOS; MARTINS, 2006). Em outro trabalho, realizado com 168 indivíduos com SD e DI, os pesquisadores observaram que $100 \%$ dos pacientes com SD apresentaram sobrepeso e obesidade, com prevalência de baixo peso em pacientes com DI (MEDONÇA; MARIANO; VIEIRA; SOUTO, LIMA, ALMEIDA, 2011).

Fatores como hábitos alimentares inadequados, dificuldades de mastigação, compulsão alimentar, disfunção da glândula tireoide, capacidade físico-motora diminuída, problemas na absorção de nutrientes, estão ligados a prevalência de sobrepeso e obesidade nesse grupo (NEVES; DURÃES; BEZERRA; CAPUCHINHO; LOPES, 2015).

$A$ avaliação do estado nutricional permite identificar a condição nutricional em que uma pessoa se encontra. A antropometria (peso, estatura, circunferências corporais e as dobras cutâneas) é o método de avaliação do estado nutricional mais amplamente utilizado em estudos epidemiológicos, por apresentar baixo custo e aplicabilidade universal. A partir desta, é possível obter informações que subsidiem condutas para o adequado cuidado nutricional e clínico do indivíduo (ACUNÃ; CRUZ, 2004).

$\mathrm{Na}$ avaliação do estado nutricional de um indivíduo, o índice da massa corporal (IMC) e o percentual de gordura corporal obtido a partir das dobras cutâneas são bons indicadores de gordura corporal (FREIRE; GORLA, 2014). As circunferências são medidas corporais utilizadas para complementar a avaliação da composição corporal, 
http://dx.doi.org/10.5902/1984686X35273

sem definir os compartimentos corporais de massa gorda, massa muscular e tamanho ósseo. A circunferência da cintura é uma medida amplamente utilizada para avaliar o índice de deposição de gordura abdominal e, indiretamente, estimar o risco de doença cardiovascular e metabólica (DUARTE, 2019). As dobras cutâneas permitem, de modo não invasivo, estimar a gordura corporal e caracterizar a distribuição da gordura subcutânea. A dobra cutânea tricipital (DCT), quando combinada com a circunferência do braço, estima a massa muscular através da aplicação de fórmulas como a circunferência muscular do braço (CMB) (ACUNÃ; CRUZ, 2004).

Diante da necessidade e da relevância do tema, o objetivo deste estudo foi identificar a composição corporal de alunos adultos com deficiência intelectual e síndrome de Down de uma instituição pública de ensino do município de Porto Velho-RO, por meio de medidas antropométricas sinalizadoras para o risco de comorbidades cardiovasculares e metabólicas ligado a deposição de gordura corporal.

\section{Metodologia}

Trata-se de um estudo do tipo transversal, com análise quantitativa dos dados. Segundo Fronteira (2013), o estudo transversal analisa a relação entre a frequência da doença e as características da população estudada, permitindo conhecer a prevalência das doenças e de fatores associados. É um estudo considerado relativamente rápido de executar e amplamente utilizado.

A pesquisa foi realizada na Escola Estadual de Ensino Especial, no município de Porto Velho-RO, Brasil. Trata-se de amostragem não probabilística por conveniência, composta pelos alunos dos quais se dispunha durante a pesquisa (VIEIRA, 1980). A escola atende alunos do $2^{\circ}$ ao $5^{\circ}$ ano, com faixa etária de 14 a 59 anos, contendo, em média, 80 alunos.

A inclusão dos alunos com DI no estudo ocorreu a partir das respostas dos pais ou responsáveis ao convite de participação e da assinatura dos termos de consentimento livre e esclarecido. A coleta de dados iniciou-se a partir do acesso aos laudos médicos, arquivados nas pastas dos alunos na secretaria da escola. Nesses laudos, os alunos eram classificados conforme os critérios do CID 10 - F70 (DI leve), F71 (DI moderado) e F72 (DI grave).

Com bases nos laudos, foram selecionados como participantes do estudo alunos com SD e DI em grau leve e moderado. Os alunos classificados como DI grave não 
http://dx.doi.org/10.5902/1984686X35273

permitiram o toque corporal para aferição de medidas antropométricas, portanto, não foram incluídos na pesquisa. Nesse grupo não foi constatada nenhuma síndrome associada a DI. Todos os alunos eram adultos, na faixa etária de 19 a 54 anos. Não participaram do estudo alunos indígenas, quilombolas, deficientes físicos, autistas, gestantes, nutrizes, aqueles cujos responsáveis não aceitaram assinar o termo de consentimento livre e esclarecido, os que não responderam ao inquérito alimentar e os alunos que não concordaram em participar do estudo.

Foram realizadas as aferições das variáveis antropométricas de peso e altura em uma balança eletrônica marca "Líder", com capacidade máxima de 200 kg e precisão de 50 g. Para aferição da estatura, foi utilizado o Estadiômetro Vertical, marca "Altura Exata", com capacidade máxima de $213 \mathrm{~cm}$, conforme o Manual de orientações para a coleta e análise de dados antropométricos em serviços de saúde, do Sistema de Vigilância Alimentar e Nutricional (SISVAN) (BRASIL, 2011). Para a aferição das dobras cutâneas (bicipital, tricipital, subescapular e suprailíaca), utilizou-se o plicômetro clínico Cescorf®, avaliadas conforme os passos descritos por Rosa (2012) e apresentados no Quadro 1.

Quadro 1 - Métodos de aferição para dobras

\begin{tabular}{|c|l|}
\hline Dobra & \multicolumn{1}{c}{ Procedimento } \\
\hline 1. Mensurar a dobra com o paciente de pé, com braços relaxados e \\
estendidos ao longo do corpo. \\
2. Padronizando o lado direito para medição e mantido nas demais medições; \\
3. Identificar, medir e marcar o local da dobra. Segurar firmemente a dobra, \\
entre o polegar e o indicador da mão esquerda, 1cm acima do local a ser \\
medido. \\
4.Destacar a dobra de modo a assegurar que o tecido muscular não tenha \\
sido pinçado. \\
5. Colocar as hastes do adipômetro perpendiculares à dobra e soltar a \\
pressão das hastes lentamente. \\
6. Manter a dobra pressionada durante a aferição. \\
7. Tomar as medições das dobras 4 segundos após a pressão ter sido \\
aplicada (Repetindo por 3 vezes para realizara média). \\
8. Abrir as hastes do adipômetro para removê-lo do local e fechá-lo.
\end{tabular}


http://dx.doi.org/10.5902/1984686X35273

Quadro 2 - Métodos de aferição para dobras

\begin{tabular}{|c|c|}
\hline Dobra & Procedimento \\
\hline Bicipital & $\begin{array}{c}\text { Aferição feita com a palma da mão voltada para fora; marcar o local da medida } \\
\text { 1cm acima do local marcado na dobra tricipital na parte anterior do braço. }\end{array}$ \\
\hline Subescapular & $\begin{array}{c}\text { Mensurar ao longo da linha natural da pele, logo abaixo do ângulo inferior da } \\
\text { escápula, com adipômetro aplicado 1 cm abaixo dos dedos, estando o } \\
\text { indivíduo com braços e ombros relaxados. }\end{array}$ \\
\hline Supra ilíaca & $\begin{array}{c}\text { Mensurada na linha média axilar, com o dedo indicador logo acima da crista } \\
\text { ilíaca, na posição diagonal, ou seja, seguindo a linha de clivagem natural da } \\
\text { pele no lado direito do indivíduo. }\end{array}$ \\
\hline
\end{tabular}

Fonte: Rosa (2012) p. 39-43.

Os resultados do Índice de massa corporal (IMC = peso/altura²) foram classificados de acordo com os pontos de corte estabelecidos pela OMS (2004), apresentados no Quadro 2.

Quadro 3 - Classificação do IMC

\begin{tabular}{|c|c|}
\hline Ponto de Corte & Classificação \\
\hline$<16 \mathrm{~kg} / \mathrm{m}^{2}$ & Magreza grau III \\
\hline $16-16,9 \mathrm{~kg} / \mathrm{m}^{2}$ & Magreza grau II \\
\hline $17-18,5 \mathrm{~kg} / \mathrm{m}^{2}$ & Magreza grau I \\
\hline $18,5-24,9 \mathrm{~kg} / \mathrm{m}^{2}$ & Eutrofia \\
\hline $25,0-29,9 \mathrm{~kg} / \mathrm{m}^{2}$ & Sobrepeso \\
\hline $30,0-34,9 \mathrm{~kg} / \mathrm{m}^{2}$ & Obesidade grau I \\
\hline $35,0-39,9 \mathrm{~kg} / \mathrm{m}^{2}$ & Obesidade grau II \\
\hline$\geq 40,0 \mathrm{~kg} / \mathrm{m}^{2}$ & Obesidade grau III \\
\hline
\end{tabular}

Fonte: UNICAMP (2003).

O índice de percentual (\%) de gordura corporal foi obtido pela conversão da soma das quatro dobras cutâneas (tríceps, bíceps, subescapular e supra ilíaca) em percentual de gordura, de acordo com Durnin e Wormersley (1967 apud REZENDE et al., 2006), classificado de acordo com Lohman (1992).

Com a circunferência do braço (CB) e a dobra cutânea triciptal (DCT), foi calculada a circunferência muscular do braço $(\mathrm{CMB})$ pela fórmula: $\mathrm{CMB}=\mathrm{CB}-(3,14 \mathrm{X} \mathrm{DCT} / 10)$, classificada pelo percentual de adequação em relação à mediana do National Health and Nutrition Examination Survey (NHANES), conforme o Quadro 3. 
http://dx.doi.org/10.5902/1984686X35273

Quadro 4 - Classificação de risco nutricional por medidas braquial

\begin{tabular}{|c|c|}
\hline Ponto de Corte & Classificação \\
\hline \multicolumn{2}{|c|}{ CB e PCT } \\
\hline$<70 \%$ & Desnutrição grave \\
\hline 70 a $80 \%$ & Desnutrição moderada \\
\hline 80 a $90 \%$ & Desnutrição leve \\
\hline 90 a $110 \%$ & Eutrofia \\
\hline 110 a $120 \%$ & Sobrepeso \\
\hline$>120 \%$ & Obesidade \\
\hline \multicolumn{2}{|c|}{ CMB } \\
\hline$<70 \%$ & Desnutrição grave \\
\hline 70 a $80 \%$ & Desnutrição moderada \\
\hline 80 a $90 \%$ & Desnutrição leve \\
\hline 90 a $110 \%$ & Eutrofia \\
\hline
\end{tabular}

Fonte: UNICAMP (2003).

A circunferência da cintura (CC) e a relação cintura-quadril (RCQ) foram classificadas segundo a Organização Mundial da Saúde - OMS 2000 (OMS, 2004). conforme o Quadro 4.

Quadro 5 - Classificação da circunferência da cintura e relação cintura/quadril

\begin{tabular}{|c|}
\hline Circunferência da Cintura \\
\hline Risco aumentado: $\geq 94 \mathrm{~cm}$ para homens $\mathrm{e} \geq 80 \mathrm{~cm}$ para mulheres; \\
Risco muito aumentado: $\geq 102 \mathrm{~cm}$ para homens $\mathrm{e} \geq 88 \mathrm{~cm}$ para mulheres \\
\hline Relação circunferência/quadril \\
Risco: $>1$ para homens \\
Risco: $>0,85$ para mulheres
\end{tabular}

Fonte: UNICAMP (2003).

Todas as classificações das medidas antropométricas foram realizadas com o auxílio do programa Nutrilife 9.9, conforme $^{1}$ exemplificado na Figura 1:

Figura 1 - Exemplo de classificação do \% de Gordura corporal

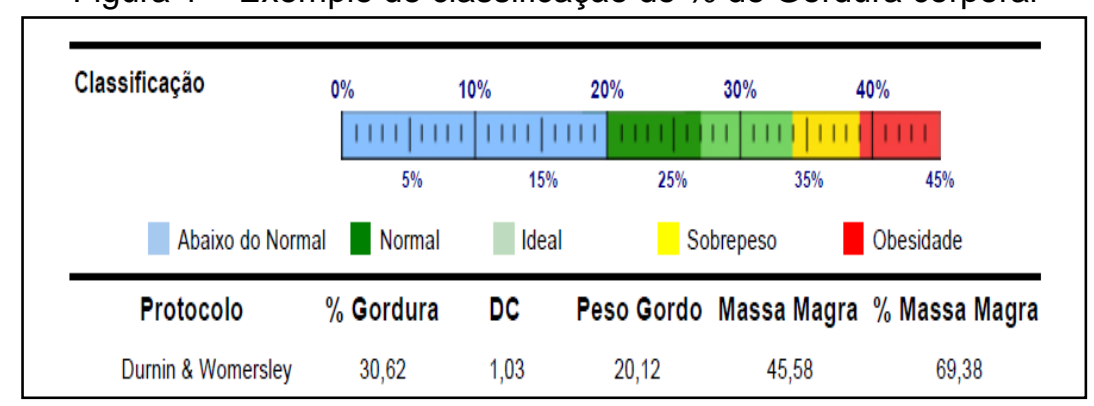

Fonte: NUTRILIFE 9.9 (2018). 
http://dx.doi.org/10.5902/1984686X35273

O armazenamento e tabulação dos dados foram realizados no programa Excel Office 2010 e para análise estatística foram utilizados os softwares: SPSS V20, Minitab 16 e Excel Office 2010. O nível de significância para este trabalho foi estabelecido em 0,05\%, com intervalos de confiança de $95 \%$; foram utilizados os testes paramétricos T-Student e Qui-Quadrado (VIEIRA, 1991).

Para a realização da pesquisa ${ }^{2}$, foram seguidas as recomendações éticas da Resolução 466, de 12 de dezembro de 2012, com disponibilização do Termo de Consentimento Livre e Esclarecido.

\section{Resultados e Discussão}

A princípio, foram distribuídos 60 termos de consentimento livre e esclarecido; destes, 21 não retornaram e 39 retornaram assinados pelos pais ou responsáveis. Dentre os que retornaram assinados, 05 foram classificados como DI grave e não permitiram o toque físico para avaliação antropométrica. $\mathrm{O}$ avaliador físico, neste grupo de pessoas, deve considerar a probabilidade de rejeição aos procedimentos, caso 0 avaliado apresente contraturas, inquietação, não compreenda as instruções ou tenha medo do equipamento (VERSTRAELEN et al., 2009).

Nesse cenário, a pesquisa foi conduzida com 34 alunos, em idade de 19 a 54 anos, sendo 21 do gênero masculino e 13 do feminino. Dentre estes, 85\% (29) foram diagnosticados com deficiência intelectual (DI) e 15\% (05) com síndrome de Down (SD). A análise quantitativa dos dados comparativos entre DI e SD, no que se refere à média, mediana, desvio, padrão, valores máximos e mínimos, intervalo de confiança e p-valores resultantes do teste T-Student estão apresentados na Tabela 01.

Verificou-se que os alunos com DI eram mais altos que os alunos com SD, com média de 1,64m (0,11DP) e 1,51m (0,06DP) ( $\mathrm{p}$-valor 0,017 - probabilidade estatística) respectivamente. Nota-se que a média do IMC é maior em alunos com SD, comparada aos alunos com DI, com média de $34,3 \mathrm{~kg} / \mathrm{m}^{2}$ (6,2 DP - desvio padrão) e $26,5 \mathrm{Kg} / \mathrm{m}^{2}$ (5,3 DP) (p-valor $=0,014)$, respectivamente. No estudo de Souza et al. (2015), os indivíduos com deficiência intelectual apresentaram média de estatura de 1,68 m e IMC em 26,8 $\mathrm{kg} / \mathrm{m}^{2}$.

De acordo com Silva e Silva (2009), pessoas com deficiência intelectual apresentam peso corporal elevado. Pessoas com síndrome de Down possuem maior predisposição para o 
excesso de peso, devido ao baixo metabolismo basal e ao sedentarismo ocasionado por alterações morfológicas que dificultam a ação motora (SILVA; SANTOS; MARTINS, 2006). Nos casos em que se associa essa predisposição com o comportamento de pais que utilizam da liberdade para comer como um mecanismo de compensação pela falha genética de seus filhos se eleva o risco de sobrepeso e obesidade desde a primeira infância (GIARETTA; GHIORZI, 2009). Pessoas com deficiência intelectual possuem os mesmos requerimentos nutricionais de calorias, proteínas e micronutrientes de indivíduos saudáveis do mesmo gênero e idade. Os maus hábitos alimentares devido ao desconhecimento, o sedentarismo e o baixo poder aquisitivo, podem contribuir para o excesso de peso neste público (MONTEIRO; NETO, 2018).

Tabela 1 - Compara deficiências para variáveis quantitativas $(\mathrm{N}=34)$

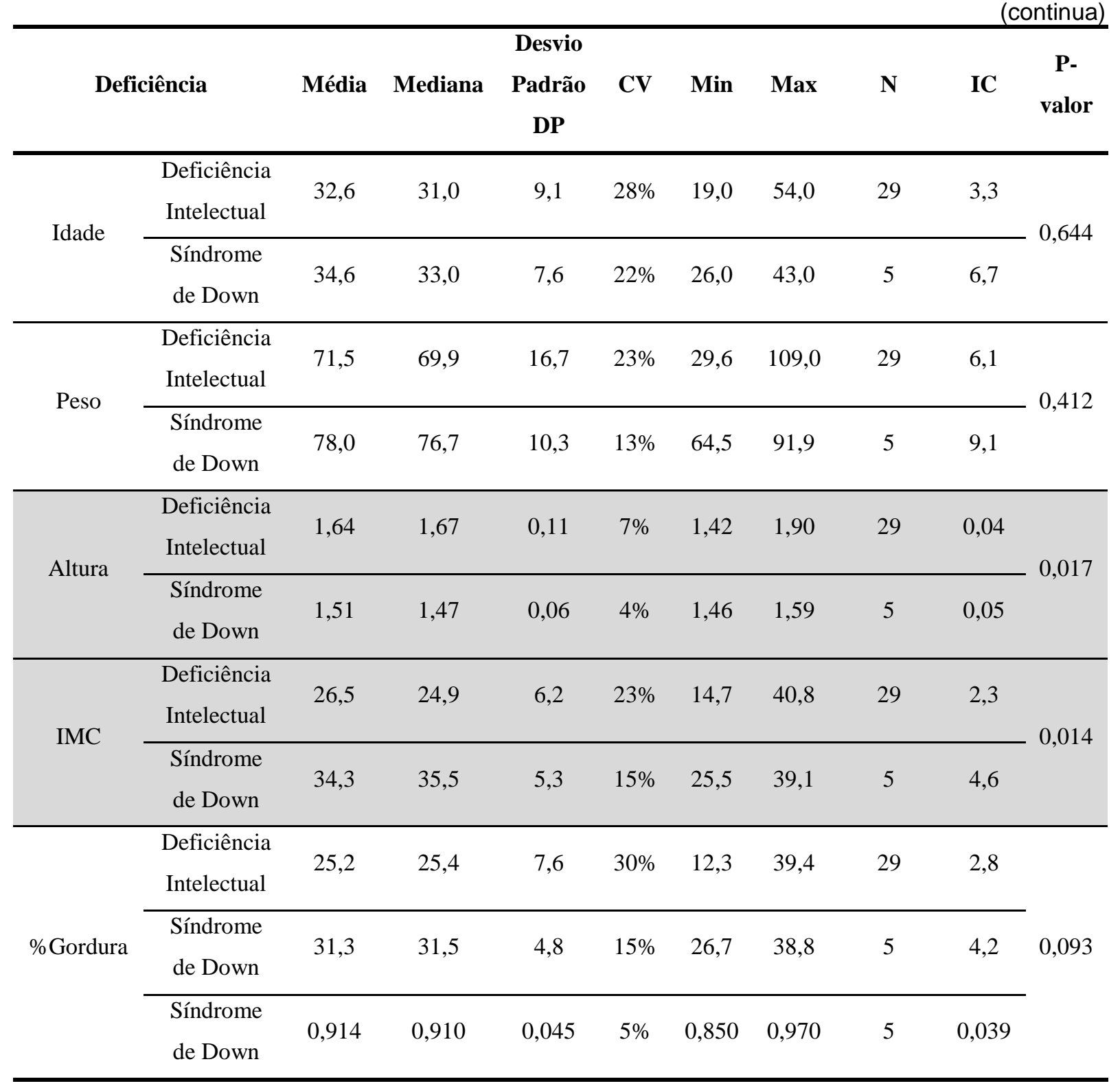


Tabela 2 - Compara deficiências para variáveis quantitativas $(\mathrm{N}=34)$

(conclusão)

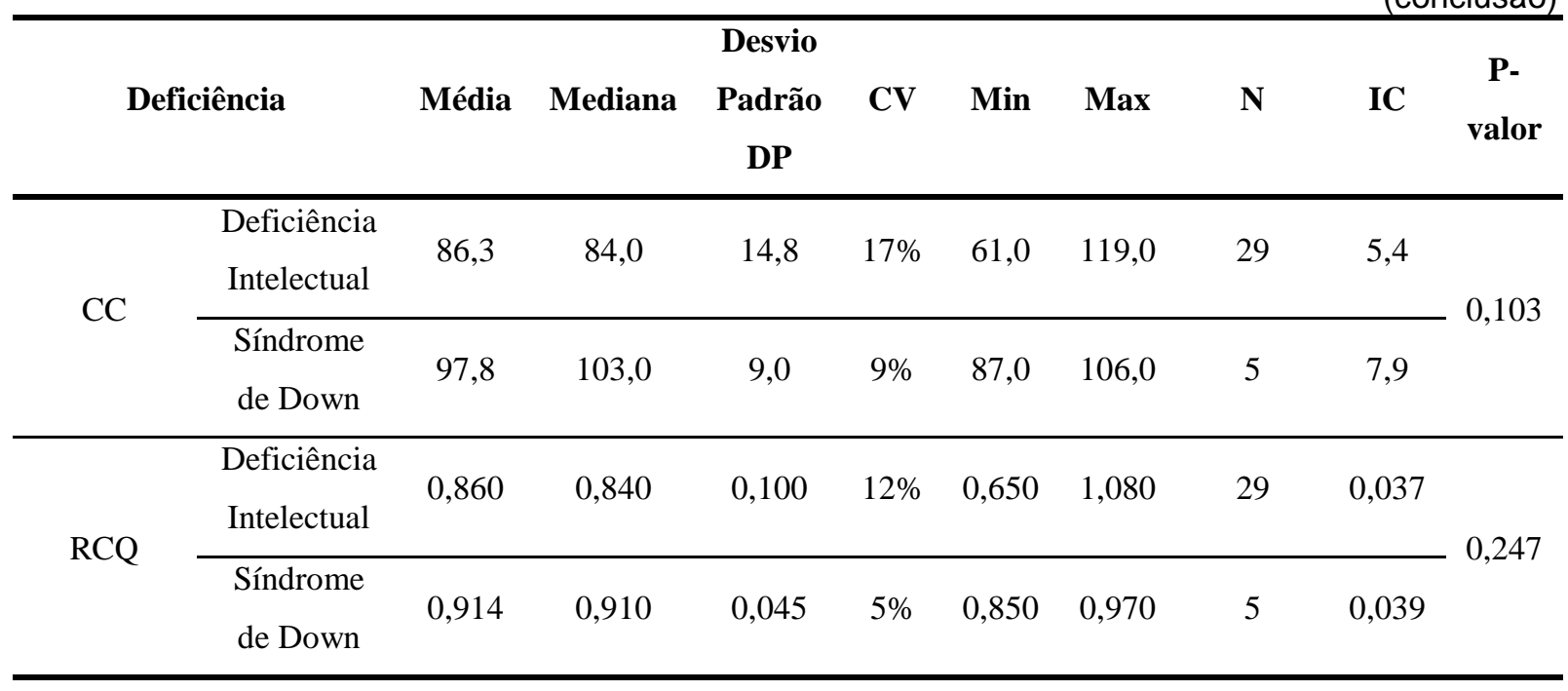

Legenda: IMC: Índice de massa corporal; CC: Circunferência da Cintura; RCQ: Relação Cintura-Quadril. Fonte: Dados da Pesquisa (2018).

A avaliação dos parâmetros antropométricos por gênero está apresentada na Tabela 2.

Tabela 3 - Compara Gênero para Variáveis Quantitativas

\begin{tabular}{|c|c|c|c|c|c|c|c|c|c|c|}
\hline \multirow{3}{*}{ Gênero } & & \multirow{3}{*}{ Média } & \multirow{3}{*}{ Mediana } & \multicolumn{3}{|l|}{ Desvio } & \multirow{3}{*}{ Max } & \multirow{3}{*}{$\mathbf{N}$} & \multirow{3}{*}{ IC } & \multirow{3}{*}{ P-valor } \\
\hline & & & & Padrão & $\mathrm{CV}$ & Min & & & & \\
\hline & & & & DP & & & & & & \\
\hline \multirow{2}{*}{ Idade } & Feminino & 35,0 & 33,0 & 10,2 & $29 \%$ & 20,0 & 54,0 & 13 & 5,5 & \multirow{2}{*}{0,276} \\
\hline & Masculino & 31,6 & 31,0 & 7,8 & $25 \%$ & 19,0 & 44,0 & 21 & 3,3 & \\
\hline \multirow{2}{*}{ Peso } & Feminino & 66,7 & 72,7 & 19,5 & $29 \%$ & 29,6 & 89,9 & 13 & 10,6 & \multirow{2}{*}{0,100} \\
\hline & Masculino & 76,0 & 71,0 & 12,6 & $17 \%$ & 54,2 & 109,0 & 21 & 5,4 & \\
\hline \multirow{2}{*}{ Altura } & Feminino & 1,54 & 1,51 & 0,09 & $6 \%$ & 1,42 & 1,68 & 13 & 0,05 & \multirow{2}{*}{$<0,001$} \\
\hline & Masculino & 1,68 & 1,68 & 0,09 & $6 \%$ & 1,47 & 1,90 & 21 & 0,04 & \\
\hline \multirow{2}{*}{ IMC } & Feminino & 28,3 & 31,9 & 8,4 & $30 \%$ & 14,7 & 40,8 & 13 & 4,6 & \multirow{2}{*}{0,688} \\
\hline & Masculino & 27,3 & 25,5 & 5,4 & $20 \%$ & 21,0 & 38,6 & 21 & 2,3 & \\
\hline \multirow{2}{*}{$\%$ Gordura } & Feminino & 31,7 & 32,8 & 5,1 & $16 \%$ & 21,4 & 38,8 & 13 & 2,8 & \multirow{2}{*}{$<0,001$} \\
\hline & Masculino & 22,7 & 22,4 & 6,7 & $30 \%$ & 12,3 & 39,4 & 21 & 2,9 & \\
\hline \multirow{2}{*}{$\mathrm{CC}$} & Feminino & 85,8 & 85,0 & 17,9 & $21 \%$ & 61,0 & 112,0 & 13 & 9,7 & \multirow{2}{*}{0,513} \\
\hline & Masculino & 89,3 & 87,0 & 12,4 & $14 \%$ & 71,4 & 119,0 & 21 & 5,3 & \\
\hline \multirow{2}{*}{ RCQ } & Feminino & 0,848 & 0,850 & 0,117 & $14 \%$ & 0,650 & 1,080 & 13 & 0,063 & \multirow{2}{*}{0,366} \\
\hline & Masculino & 0,880 & 0,870 & 0,081 & $9 \%$ & 0,740 & 1,040 & 21 & 0,035 & \\
\hline
\end{tabular}

Legenda: IMC: Índice de massa corporal; CC: Circunferência da Cintura; RCQ: Relação Cintura-Quadril. Fonte: Dados da Pesquisa (2018). 
A altura dos homens, comparada à das mulheres, foi significativamente mais elevada ( $p$-valor $<0,001$ ). As curvas de crescimento linear apresentadas no relatório da pesquisa de orçamento familiar 2008-2009 mostram que a população em geral, a partir dos 15 anos de idade, apresenta uma desaceleração do crescimento no sexo feminino, mantendo as mulheres com estatura abaixo dos homens (IBGE, 2010). Neste grupo de adultos com deficiência intelectual, esse padrão populacional se manteve.

Melville; Cooper; Mcgrother; Thorp e Collacott (2005) avaliaram dados de 1992-1993 da população de Leicestershire (condado da zona leste da Inglaterra) com SD e controle com DI. Concluíram que mulheres com SD foram mais propensas a ter sobrepeso e obesidade e os homens foram mais propensos ao sobrepeso. No grupo com SD, houve significante IMC mais elevado (29,65; 6,4DP) que o grupo controle $(27,4 ; 7,6 \mathrm{DP})$; entre homens não houve diferença estatisticamente significante (27,03; 5,25DP), comparado ao controle (26,137,7DP). Baixo peso foi prevalente no grupo com DI.

Em média, ambos os gêneros apresentaram sobrepeso de acordo com o IMC. Conforme o relatório da VIGITEL (BRASIL, 2017), a frequência média de sobrepeso ou excesso de peso na população adulta foi maior entre homens. O percentual de gordura corporal nas mulheres foi mais elevado ( $p$-valor $<0,001$ ), bem como o risco aumentado para doenças cardiovasculares, resistência insulínica e diabetes tipo II pela circunferência da cintura. Medidas antropométricas de 76 adultos, 67\% masculinos, com idade entre 19 e 72 anos portadores de DI, mostraram que o IMC e a circunferência da cintura são as opções mais viáveis de mensuração da composição corporal, quando comparadas às medidas de dobras cutâneas e impedância bioelétrica (BIA). O IMC, nesse mesmo estudo, classificou $64 \%, 36 \%$ e $18 \%$ dos pacientes em baixo peso, sobrepeso e obesidade, respectivamente. Quanto à circunferência da cintura, 22\% foram classificados com sobrepeso e $29 \%$ obesos (VERSTRAELEN; MAASKANT; KNIJFF-RAEVEN; CURFS; SCHROJENSTEIN LANTMAN, 2009).

O Gráfico 1 apresenta a distribuição da frequência de classificação do IMC por gênero. 
http://dx.doi.org/10.5902/1984686X35273

Gráfico 1 - Resultados do IMC total e por gêneros

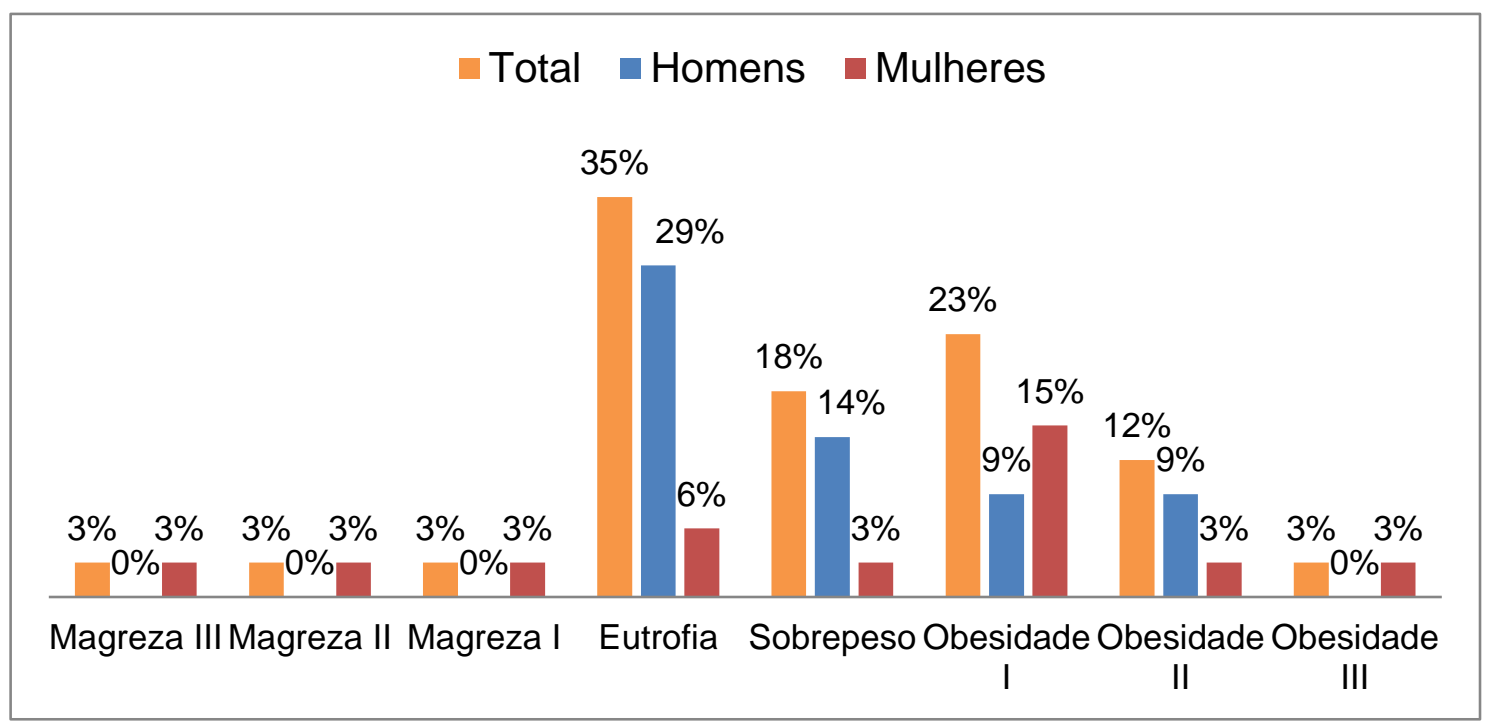

Fonte: Dados da pesquisa (2018).

Os resultados indicam obesidade grau III, magreza grau I, II e III apenas em mulheres. A soma das frequências de excesso de peso do total de avaliados foi $56 \%$. Esses resultados foram tão elevados quanto os encontrados em estudo brasileiro de população sem diagnóstico de DI (VIGITEL BRASIL, 2016), no qual 53,8\% dos entrevistados foram classificados como excesso de peso (BRASIL, 2017). No entanto, foram superiores aos encontrados por Silva e Silva (2009), no qual 37\% de pessoas com DI apresentavam sobrepeso/obesidade e foram semelhantes aos encontrados por Sari; Yilmaz; Serin; Kisa; Yesiltepe; Tokem e Rowley (2016), em estudo realizado na Turquia, com 347 adolescentes e adultos com DI. Nessa pesquisa, os autores verificaram que 52\% dos adultos tinham sobrepeso ou obesidade, de acordo com o IMC.

Portadores de necessidades especiais podem viver em condições de limitações sociais e cognitivas que comprometem a aptidão física (MELVILLE; COOPER; MCGROTHER; THORP; COLLACOTT, 2005). Os mesmos precisam de estímulos e do apoio familiar para evitar o excesso de gordura corporal e preservar a massa magra. A análise comparativa das classificações nutricionais dos compartimentos de gordura e massa muscular entre os dois grupos de alunos está exposta na Tabela 3. 
Tabela 4 - Comparativo das variáveis indicadoras de gordura e de massa muscular para o tipo de deficiência

\begin{tabular}{|c|c|c|c|c|c|c|c|c|}
\hline & & \multicolumn{2}{|c|}{$\begin{array}{l}\text { Deficiência } \\
\text { Intelectual }\end{array}$} & \multicolumn{2}{|c|}{$\begin{array}{c}\text { Síndrome de } \\
\text { Down }\end{array}$} & \multicolumn{2}{|c|}{ Total } & \multirow[t]{2}{*}{ P-valor } \\
\hline & & $\mathrm{N}$ & $\%$ & $\mathrm{~N}$ & $\%$ & $\mathrm{~N}$ & $\%$ & \\
\hline \multirow{3}{*}{ CLASS CC } & Risco elevado & 4 & $13,8 \%$ & 0 & $0,0 \%$ & 4 & $11,8 \%$ & \multirow{3}{*}{0,046} \\
\hline & Risco muito elevado & 7 & $24,1 \%$ & 4 & $80,0 \%$ & 11 & $32,4 \%$ & \\
\hline & Sem risco & 18 & $62,1 \%$ & 1 & $20,0 \%$ & 19 & $55,9 \%$ & \\
\hline \multirow{3}{*}{$\begin{array}{c}\text { CLASS } \\
\text { CMB }\end{array}$} & Desnutrição leve & 8 & $27,6 \%$ & 0 & $0,0 \%$ & 8 & $23,5 \%$ & \multirow{3}{*}{0,246} \\
\hline & Desnutrição moderada & 3 & $10,3 \%$ & 0 & $0,0 \%$ & 3 & $8,8 \%$ & \\
\hline & Eutrofia & 18 & $62,1 \%$ & 5 & $100 \%$ & 23 & $67,6 \%$ & \\
\hline \multirow{4}{*}{ CLASS \% G } & Ideal & 9 & $31,0 \%$ & 1 & $20,0 \%$ & 10 & $29,4 \%$ & \multirow{4}{*}{0,121} \\
\hline & Normal & 9 & $31,0 \%$ & 0 & $0,0 \%$ & 9 & $26,5 \%$ & \\
\hline & Obesidade & 2 & $6,9 \%$ & 2 & $40,0 \%$ & 4 & $11,8 \%$ & \\
\hline & Sobrepeso & 9 & $31,0 \%$ & 2 & $40,0 \%$ & 11 & $32,4 \%$ & \\
\hline \multirow{4}{*}{$\begin{array}{c}\text { CLASS } \\
\text { RCQ }\end{array}$} & Risco alto & 6 & $20,7 \%$ & 4 & $80,0 \%$ & 10 & $29,4 \%$ & \multirow{4}{*}{0,045} \\
\hline & Risco baixo & 7 & $24,1 \%$ & 0 & $0,0 \%$ & 7 & $20,6 \%$ & \\
\hline & Risco moderado & 10 & $34,5 \%$ & 0 & $0,0 \%$ & 10 & $29,4 \%$ & \\
\hline & Risco muito alto & 6 & $20,7 \%$ & 1 & $20,0 \%$ & 7 & $20,6 \%$ & \\
\hline \multirow{6}{*}{$\begin{array}{c}\text { CLASS } \\
\text { DCT }\end{array}$} & Desnutrição grave & 9 & $31,0 \%$ & 0 & $0,0 \%$ & 9 & $26,5 \%$ & \multirow{6}{*}{0,576} \\
\hline & Desnutrição leve & 5 & $17,2 \%$ & 1 & $20,0 \%$ & 6 & $17,6 \%$ & \\
\hline & Desnutrição moderada & 2 & $6,9 \%$ & 0 & $0,0 \%$ & 2 & $5,9 \%$ & \\
\hline & Eutrofia & 6 & $20,7 \%$ & 1 & $20,0 \%$ & 7 & $20,6 \%$ & \\
\hline & Obesidade & 5 & $17,2 \%$ & 2 & $40,0 \%$ & 7 & $20,6 \%$ & \\
\hline & Sobrepeso & 2 & $6,9 \%$ & 1 & $20,0 \%$ & 3 & $8,8 \%$ & \\
\hline
\end{tabular}

Legenda: CLASS CC: Classificação da Circunferência da Cintura; CLASS CMB: Classificação da Circunferência Muscular do Braço; CLASS \% G: Classificação do Percentual de Gordura; CLASS RCQ: Classificação da Relação Cintura - Quadril; CLASS DCT: Classificação da Dobra Cutânea Tricipital. Fonte: Dados da Pesquisa (2018).

Os estudantes com SD apresentaram CC e RCQ com risco muito elevado e risco elevado, respectivamente, para doenças cardiovasculares e metabólicas. Nessas duas variáveis, houve diferença significativa entre SD e DI ( $p$-valor <0,0446; $p$-valor $<0,045$ ). No total de estudantes, houve maior frequência de risco elevado ou muito elevado para doenças cardiovasculares e metabólicas, conforme a CC (44.2\%) e RCQ (50,0\%). Outros autores, na Turquia, identificaram, em adultos com DI, que $65,5 \%$ das mulheres e $41 \%$ dos homens tinham a CC classificada no grupo de risco elevado e muito elevado, $54,5 \%$ das mulheres e $48,6 \%$ dos homens estavam em risco alto, de acordo com a RCQ. Houve correlação significativa entre os 
valores de pressão arterial sistólica e diastólica e IMC, circunferência da cintura e relação cintura- quadril nos adultos (SARI; YILMAZ; SERIN; KISA; YESILTEPE; TOKEM; ROWLEY, 2016). A massa muscular avaliada pela circunferência muscular do braço se mostrou preservada nos dois grupos de alunos, porém ressalta-se que $27,6 \%$ dos alunos com DI foram classificados com massa muscular em desnutrição leve. Sousa; Pinto; Seeber; Silva. (2015) associaram o estado nutricional e de saúde com a aptidão física de alunos adultos com DI (excluídos SD) e identificaram que a maioria estava com excesso de peso pelo IMC, excesso de gordura corporal pelas dobras cutâneas; teve-se o pior desempenho nos testes de aptidão física, quando comparados aos eutróficos. Na revisão sistemática da literatura realizada por Pestana; Barbieri; Vitório; Figuereido; Castro. (2018), foram analisados os efeitos do exercício físico para adultos com DI em todos os graus (leve a severo), de ambos os sexos e idade entre 18 e 67 anos. O período de intervenção foi de 2,5 até 9 meses e frequência semanal de uma vez até três vezes por semana. Os autores concluíram que exercícios que combinam força muscular e resistência aeróbica promovem benefícios físicos, bem-estar e qualidade de vida para adultos com DI.

$\mathrm{Na}$ Tabela 4, apresentam-se as classificações dos mesmos parâmetros de avaliação da distribuição de massa gorda e de massa muscular por gênero.

Tabela 5 - Comparativo das circunferências e dobras com o gênero

\begin{tabular}{|c|c|c|c|c|c|c|c|c|}
\hline & \multirow{2}{*}{ Classificação } & \multicolumn{2}{|c|}{ Feminino } & \multicolumn{2}{|c|}{ Masculino } & \multicolumn{2}{|c|}{ Total } & \multirow{2}{*}{ P-valor } \\
\hline & & $\mathrm{N}$ & $\%$ & $\mathrm{~N}$ & $\%$ & $\mathrm{~N}$ & $\%$ & \\
\hline \multirow{3}{*}{$\begin{array}{c}\text { CLASS } \\
\text { CC }\end{array}$} & Risco elevado & 3 & $23,1 \%$ & 1 & $4,8 \%$ & 4 & $11,8 \%$ & \multirow{3}{*}{0,052} \\
\hline & Risco muito elevado & 6 & $46,2 \%$ & 5 & $23,8 \%$ & 11 & $32,4 \%$ & \\
\hline & Sem risco & 4 & $30,8 \%$ & 15 & $71,4 \%$ & 19 & $55,9 \%$ & \\
\hline \multirow{3}{*}{$\begin{array}{c}\text { CLASS } \\
\text { CMB }\end{array}$} & Desnutrição leve & 2 & $15,4 \%$ & 6 & $28,6 \%$ & 8 & $23,5 \%$ & \multirow{3}{*}{0,640} \\
\hline & Desnutrição moderada & 1 & $7,7 \%$ & 2 & $9,5 \%$ & 3 & $8,8 \%$ & \\
\hline & Eutrofia & 10 & $76,9 \%$ & 13 & $61,9 \%$ & 23 & $67,6 \%$ & \\
\hline \multirow{4}{*}{$\begin{array}{c}\text { CLASS } \\
\% \mathrm{G}\end{array}$} & Ideal & 4 & $30,8 \%$ & 6 & $28,6 \%$ & 10 & $29,4 \%$ & \multirow{4}{*}{0,886} \\
\hline & Normal & 3 & $23,1 \%$ & 6 & $28,6 \%$ & 9 & $26,5 \%$ & \\
\hline & Obesidade & 1 & $7,7 \%$ & 3 & $14,3 \%$ & 4 & $11,8 \%$ & \\
\hline & Sobrepeso & 5 & $38,5 \%$ & 6 & $28,6 \%$ & 11 & $32,4 \%$ & \\
\hline \multirow{4}{*}{$\begin{array}{c}\text { CLASS } \\
\text { RCQ }\end{array}$} & Risco alto & 4 & $30,8 \%$ & 6 & $28,6 \%$ & 10 & $29,4 \%$ & \multirow{4}{*}{0,153} \\
\hline & Risco baixo & 1 & $7,7 \%$ & 6 & $28,6 \%$ & 7 & $20,6 \%$ & \\
\hline & Risco moderado & 3 & $23,1 \%$ & 7 & $33,3 \%$ & 10 & $29,4 \%$ & \\
\hline & Risco muito alto & 5 & $38,5 \%$ & 2 & $9,5 \%$ & 7 & $20,6 \%$ & \\
\hline
\end{tabular}


http://dx.doi.org/10.5902/1984686X35273

Tabela 6 - Comparativo das circunferências e dobras com o gênero

\begin{tabular}{|c|c|c|c|c|c|c|c|c|}
\hline & \multirow{2}{*}{ Classificação } & \multicolumn{2}{|c|}{ Feminino } & \multicolumn{2}{|c|}{ Masculino } & \multicolumn{2}{|c|}{ Total } & \multirow{2}{*}{ P-valor } \\
\hline & & $\mathrm{N}$ & $\%$ & $\mathrm{~N}$ & $\%$ & $\mathrm{~N}$ & $\%$ & \\
\hline \multirow{6}{*}{$\begin{array}{c}\text { CLASS } \\
\text { DCT }\end{array}$} & Desnutrição grave & 5 & $38,5 \%$ & 4 & $19,0 \%$ & 9 & $26,5 \%$ & \multirow{6}{*}{0,039} \\
\hline & Desnutrição leve & 2 & $15,4 \%$ & 4 & $19,0 \%$ & 6 & $17,6 \%$ & \\
\hline & Desnutrição moderada & 0 & $0,0 \%$ & 2 & $9,5 \%$ & 2 & $5,9 \%$ & \\
\hline & Eutrofia & 3 & $23,1 \%$ & 4 & $19,0 \%$ & 7 & $20,6 \%$ & \\
\hline & Obesidade & 0 & $0,0 \%$ & 7 & $33,3 \%$ & 7 & $20,6 \%$ & \\
\hline & Sobrepeso & 3 & $23,1 \%$ & 0 & $0,0 \%$ & 3 & $8,8 \%$ & \\
\hline
\end{tabular}

Legenda: CLASS CC: Classificação da Circunferência da Cintura; CLASS CMB: Classificação da Circunferência Muscular do Braço; CLASS \% G: Classificação do Percentual de Gordura; CLASS RCQ: Classificação da Relação Cintura - Quadril; CLASS DCT: Classificação da Dobra Cutânea Tricipital. Fonte: Dados da Pesquisa (2018).

Observa-se que as mulheres apresentaram maior prevalência de risco muito elevado para desenvolver doenças cardiovasculares e metabólicas, pela CC. Resultados semelhantes foram encontrados por Silva; Gomes Filho; Silva; Fernandes Filho. (2009), sendo o maior acúmulo de gordura central em mulheres. Souza; Rodrigues; Ferreira. (2013) identificaram $100 \%$ das mulheres com a circunferência da cintura elevada em relação aos homens, em média de $85,8 \mathrm{~cm}$ nas mulheres e $89,3 \mathrm{~cm}$ nos homens.

A Organização Mundial da Saúde (OMS) preconiza que, além do IMC, devem ser avaliadas as circunferências da cintura (CC) e do quadril (CQ), pois o aumento de depósito de gordura abdominal é um indicador sensível para doenças cardiovasculares e metabólicas (GROSSL; KARASIK; LIMA, 2010). Rezende; Rosado; Priore. (2010) ressaltam que essas medidas, apesar de não indicarem quantidade e distribuição de gordura corporal, possuem importância na avaliação do risco de mortalidade. De acordo com Picon, Leitão; Gerchman; Azevedo; Silveiro; Gross; Canani. (2007, p. 444) "a obesidade androgênica está associada ao maior fator de risco de doenças coronarianas, diabetes mellitus tipo 2 e hipertensão arterial sistêmica, em ambos os sexos".

A circunferência muscular do braço avalia a reserva de tecido muscular sem correção da massa óssea (UNICAMP, 2003). Percebe-se que a maioria 67,6\% ( $n=23)$ está com massa muscular preservada.

Quanto ao percentual de gordura, observa-se que a maior frequência de sobrepeso foi nas mulheres, não havendo diferença significativa entre os gêneros ( $p$-valor 0,886 ).

$\mathrm{Na}$ relação cintura-quadril (RCQ) observa-se que a maioria dos alunos apresentam risco para o desenvolvimento de doenças cardiovasculares, com prevalência de risco 
moderado e alto para os homens e risco muito alto para as mulheres. Esses dados são preocupantes, pois a relação cintura-quadril é indicadora de adiposidade visceral, sendo umas das medidas utilizadas na avaliação de risco cardiovascular (GIUNTOLI; OUTA; MONTEIRO; MONOMI; MORAES; STULBACH, 2012).

A prega cutânea tricipital afere a reserva de gordura corporal do braço (UNICAMP, 2003). Nota-se a prevalência de desnutrição grave entre as mulheres - este resultado confirma a distribuição centralizada no abdômen - e os homens com o maior índice de obesidade por esta medida antropométrica. Sendo essas medidas comparadas com referências geradas de populações saudáveis, nos pontos de corte de classificação para homens adultos ativos os valores de porcentagem de gordura são muito menores que para as mulheres. Na população do presente estudo, o parâmetro de PCT requer associação com o grau de deficiência e de atividade física.

Pessoas com SD possuem, em sua morfologia, propensão ao ganho de peso e maior reserva de gordura. Portanto, a orientação nutricional apropriada é essencial para evitar o ganho de peso indesejado, o qual provoca limitações físicas e sociais. O ganho excessivo de peso em pessoas com SD e DI está ligado a vários fatores, como a alimentação inadequada e falta de atividade física (SILVA et al., 2006). A promoção da saúde da pessoa com deficiência intelectual depende da articulação entre diferentes setores governamentais e da sociedade civil (TOMAZ et al., 2016).

As políticas e intervenções para esse grupo de pessoas devem priorizar, além do indivíduo, a qualidade de vida da família (QdVF). Segundo a Organização Mundial de Saúde - OMS, a qualidade de vida é definida sobre domínios subjetivos e consiste na "percepção do indivíduo de sua posição na vida no contexto da cultura e sistema de valores nos quais ele vive e em ralação aos seus objetivos, padrões e preocupações". Os domínios objetivos estão relacionados a fatores como educação, nível sócio-econômico, características físicas, sociais e culturais que influenciam na autopercepção da qualidade de vida (RONCA; BLASCOVI-ASSIS, 2017).

Os estudos de QdVF interessam aos investigadores pelo seu potencial avaliativo e intervencional junto às famílias. Vale destacar que existem instrumentos validados para avaliar a QdVF de pessoas com deficiência intelectual, como o Family Quality of Life Survey (FQOLS-2006) e o Beach Center FQOL Survey (CORREIA; SEABRA-SANTOS, 2018). 
Uma revisão sistemática da literatura, conduzida por Correia e Seabra- Santos (2018), sobre a avaliação da qualidade de vida familiar (QdVF) de pessoas com DI, identificou que a ausência de saúde física interfere mais na qualidade de vida da família como um todo do que a existência de um membro com deficiência intelectual. $\mathrm{O}$ excesso de peso é causa de alterações metabólicas, dificuldades respiratórias e do aparelho locomotor, portanto, o diagnóstico e o tratamento ideal devem garantir qualidade de vida e prevenir a ocorrência de complicações habituais e severas (GALDINA, 2012).

A SD e DI por si já comprometem diversos segmentos da saúde psicomotora que requerem oportunidades diversificadas de atividades e práticas sociais promovidas pela família e por profissionais no campo da educação especial (DELGADO et al., 2019).

\section{Conclusão}

Foram analisadas as medidas antropométricas de peso, altura, circunferências e dobras cutâneas. Verificou-se que os alunos com DI eram mais altos do que os alunos com SD. No entanto, estes últimos apresentaram o índice de massa corporal mais elevada. O excesso de peso encontrado pelo IMC foi superior ao identificado na população brasileira. Quanto ao risco de doenças cardiovasculares e metabólicas, pelas medidas de circunferência da cintura e relação cintura-quadril, os estudantes portadores de SD foram classificados com risco muito elevado e muito alto, respectivamente. Por este indicador, o risco foi mais prevalente entre as mulheres, assim como houve maior frequência de sobrepeso pelo percentual de gordura corporal. A massa muscular, avaliada pela $\mathrm{CMB}$, apresentou maior prevalência de ausência de risco, embora uma parcela de alunos com DI foi classificada em desnutrição leve por este indicador.

Tanto a desnutrição como o excesso de peso devem ser levados em consideração ao serem realizadas pesquisas e ações educativas multidisciplinares dirigidas às pessoas com DI e SD, aos cuidadores e aos familiares.

\section{Referências}

AAIDD - American Association on Intellectual and Developmental Disabilities. Definitions. Accessed May 12, 2019, from http://www.aaidd.org/content_100.cfm?navlD=21.

AAMR - American Association on Mental Retardation. Retardo mental: definição, classificação e sistemas de apoio. 10 ed. Porto Alegre: Artmed, 2006.

ABESO- Associação Brasileira para o Estudo da Obesidade e da Síndrome Metabólica. 
http://dx.doi.org/10.5902/1984686X35273

Diretrizes brasileiras de obesidade 2009/2010. 3 ed. Itapevi, SP: AC Farmacêutica, 2009.

ACUNÃ, Kátia; CRUZ, Thomaz. Avaliação do estado nutricional de adultos e idosos e situação nutricional da população brasileira. Arquivo Brasileiro de Endocrinologia e Metabologia, v. 48, n. 3, p. 345- 365, Junho, 2004.

BERTAPELLI, Fábio; GORLA, José Irineu; COSTA Leonardo Trevizan; FREIRE, Fábia. Composição corporal em jovens com síndrome de Down: aspectos genéticos, ambientais e fisiológicos. Arq. Ciênc. Saúde UNIPAR, Umuarama, v. 15, n. 2, p. 197-207, maio/ago. 2011.

BRASIL. Ministério da Saúde. Secretaria de Atenção à Saúde. Departamento de Atenção Básica. Orientações para a coleta e análise de dados antropométricos em serviços de saúde: Norma Técnica do Sistema de Vigilância Alimentar e Nutricional - SISVAN. Ministério da Saúde, Brasília, 2011.

BRASIL, Ministério da Saúde. VIGITEL BRASIL 2016: estimativas sobre frequência e distribuição sócio- demográfica de fatores de risco e proteção para doenças crônicas nas capitais dos 26 estados brasileiros e no Distrito Federal em 2016. Brasília, 2017.

BAGNI, Ursula Viana; OLIVEIRA, Annamary Nascimento; PINTO, Cristiane Jordânia; SANTOS, Letícia Sabino; ARAÚJO, Joyce Samara Marques Oliveira. Aconselhamento nutricional oferecido a crianças e adolescentes com deficiência. Revista Brasileira de Promoção a Saúde, Fortaleza, v. 28, n.3, p. 418-426, jul./set., 2015.

CORREIA, Raquel Alveirinho; SEABRA-SANTOS, Maria João. Qualidade de vida familiar na deficiência intelectual: revisão sistemática de estudos. Psic.: Teor. e Pesq., Brasília, v. 34, e34414, 2018.

DELGADO, Isabelle Cahino; BARBOSA, Talita Maria Monteiro Farias; MACÊDO, Bruna Samyres Oliveira; LIMA, Cynderella Karla Moraes; RÉGIS, Mariane Sousa; LIMA, Ivonaldo Leidson Barbosa; ALVES Giorvan Ânderson dos Santos. Estratégias de letramento voltadas à intervenção fonoaudiológica em pessoas com síndrome de Down. Revista Educação Especial | v. 32, 2019.

DUARTE, Antonio Cláudio Goulart. Semiologia nutricional. 1. ed. Rio de Janeiro: Atheneu, 2019, p. 63.

FREIRE, Fábia; COSTA, Leonardo Trevisan; GORLA, José Irineu. Indicadores de obesidade em jovens com síndrome de Down. Motricidade, v. 10, n. 2, p. 2-10, 2014.

FRONTEIRA, Inês. Estudos observacionais na era da medicina baseada na evidência: breve revisão sobre a sua relevância, taxonomia e desenhos. Acta Medica Portuquesa, v. 26, p.161-170, 2013.

GALDINA Aniele Pacheco. A importância do cuidado nutricional na síndrome de Down. Dissertação de especialização em nutrição clínica - Universidade do Extremo Sul Catarinense - UNESC. Criciúma, Santa Catarina, 2012. 
GIARETTA, Andréa; GHIORZI, Angela da Rosa. O ato de comer e as pessoas com síndrome de Down. Revista Brasileira de Enfermagem, maio-jun, v. 62, n. 3, p. 480-4, Brasília, 2009.

GIUNTOLI, Ana Beatriz Brandão; OUTA, Kátia; MONTEIRO, Patrícia Maria Akagui.; MONOMI, Priscila Yumi; MORAES, Stephanie Zapparoli de; STULBACH, Tamara. Comparação entre o índice de massa corpórea e porcentagem de gordura e análise da relação cintura/quadril em desportistas de uma academia de São Paulo. EFD eportes.com, Revista Digital. Buenos Aires, v. 17, n. 171, Agosto, 2012.

GONÇALVES, Marcia Ribeiro. Perfil nutricional de indivíduos com síndrome de Down. Monografia. Centro Universitário de Brasília. 2014.

GROSSL, T.; KARASIAK, F.; LIMA, L. R. A de. Relação entre a gordura corporal e indicadores antropométricos em adultos frequentadores de academia. Motricidade, São Paulo, v. 6, n. 2, p. 35-45, mar, 2010.

IBGE- Instituto Brasileiro de Geografia e Estatística. Educa jovem - Conheça o Brasil populações - Pessoas com deficiência, 2010. Disponível em:

https://educa.ibge.gov.br/jovens/conheca-o-brasil/populacao/20551-pessoas-comdeficiencia.html. Acesso em: 28/03/2020.

IBGE - Instituto Brasileiro de Geografia e Estatística. Pesquisa de orçamento familiar 2008-2009: antropometria, estado nutricional de crianças, adolescentes e adultos no Brasil. Rio de Janeiro, 2010.

LOHMAN, T.G. Advances in body composition assessment: current issues in exercise series. Champaign, IL: Human kinetics, 1992.

MAHAN, L. Kathleen; STUM Sylvia Escott; RAYMOND Janice L. Alimentos, nutrição e dietoterapia. 13aㅡ ed. Rio de Janeiro: Elsevier, 2012. 1227p.

MEDONÇA, Bruna Dias; MARIANO, Fernanda Geórgia Werneck; VIEIRA, Laís de Jesus; SOUTO, Larissa Lafit Lucchesi; LIMA, Vanessa Calazães Campos; ALMEIDA, Nízia Araújo. Perfil nutricional de pacientes portadores de síndrome de Down, paralisia cerebral e retardo mental atendido no PAOPE da Univale, no período de agosto de 2010 a setembro de 2011. Monografia. Universidade Vale do Rio Doce, 2011.

MELVILLE C.A.; COOPER S.A.; MCGROTHER, C.W.; THORP, C. F.; COLLACOTT R. Obesity in adults with Down syndrome: a case-control study. Journal of Intellectual Disability Research, v. 49, part 2, p. 125-133, 2005.

MONTEIRO, Lorena Ligia Lima; NETO, Celso Lourenço Arruda. Estado nutricional e consumo alimentar de pessoas com deficiência intelectual. Revista da Universidade Vale do Rio Verde, v. 16, n.12, ago./dez., p. 1-10, 2018.

NEVEZ, Lilian Ferreira; DURÃES, Grayce Laiz Lima Silveira; BEZERRA, Kátia Francielly; CAPUCHINHO, Laura Croline Ferreira Mendes; LOPES, Wanessa Casteluber. Hábitos alimentares: sua influência no índice de massa corporal (IMC) em portadores de síndrome 
http://dx.doi.org/10.5902/1984686X35273

de Down. Caderno de Ciências Agrárias, Montes Claros, v. 7, n. 2, p. 40-44, mai/ago, 2015.

OMS. Obesidade:prevenindo e controlando a epidemia global. Relatório da consultoria da OMS. Tradução: Andréa Favano;revisão científica: Sérgio setsuo Maeda. São Paulo: Roca, 2004.

PESTANA, Mayara Borowskke; BARBIERI, Fabio Ausgusto; VITÓRIO, Rodrigo; FIGUEIREDO, Gabriella Andreeta; CASTRO, Eliane Mauerberg. Efeitos do exercício físico para adultos com deficiência intelectual: uma revisão sistemática. J Physical Edu, v. 29, e2920, p. 1-16, 2018.

PICON, Paula Xavier; LEITÃO, Cristiane Bauerman; GERCHMAN, Fernando; AZEVEDO, Mirela Jobim de; SILVEIRO, Sandra Pinho; GROSS, Jorge Luiz; CANANI, Luís Henrique. Medida da cintura e razão cintura/quadril e identificação de situações de risco cardiovascular: estudo multicêntrico em pacientes com diabetes melito Tipo 2. Arquivo Brasileiro de Endocrinologia e Metabologia, v. 51, n.3, p. 443- 449, 2007.

REZENDE, Fabiane Aparecida Canaan; ROSADO, Lina Enriqueta Paez Lima; PRIORE, Silvia Eloiza. Aplicabilidade de equações na avaliação da composição corporal da população brasileira. Rev. Nutr., Campinas, 19 (3): 357-367, mai/jun., 2006.

RONCA, Roberta Pasqualucci; BLASCOVI-ASSIS, Silvana Maria. QUALIDADE DE VIDA DE CUIDADORES DE PESSOAS COM SÍNDROME DE DOWN: REVISÃO DE LITERATURA. Cadernos de Pós-Graduação em Distúrbios do Desenvolvimento, São Paulo, v.17, n.1, p. 26-38, 2017.

ROSA, Glorimar. Avaliação nutricional do paciente hospitalizado - uma abordagem teórico-prática. Rio de Janeiro - RJ: Guanabara Koogan, 2012.

SARI, Hatice Yildirim; YILMAZ, Medine; SERIN, Elif; KISA, Sezer Secgin; YESILTEPE, Ozlem; TOKEM, Yasemin; ROWLEY, Helen. Obesidade e hipertensão em adolescentes e adultos com deficiência intellectual. Acto Paulisa de enfermagem, São Paulo, vol. 29, núm. 2, marzo-abril, pp.169-177, 2016.

SILVA, Domingos Lopes da; SANTOS, José Augusto Rodrigues dos; MARTINS, Carla Ferreira. Avaliação da composição corporal em adultos com síndrome de Down.

Arquivos de Medicina, v.10, n.4, p. 103-10, 2006.

SILVA-JUNIOR, Carlos Alves da; TORNELLO, Maria Georgina Marques; GORLA, José Irineu; CALEGARI, Décio Roberto. Musculação para um aluno com síndrome de Down e aumento da resistência muscular localizada. Rev. Digital, 2007.

SILVA, Rudney da, SILVA, Gabriel Pires. Características antropométricas e nutricionais de pessoas com deficiência mental. Fitness Performacé Journal, v.8, n. 2, p.130-5, marabr, 2009.

SILVA, Naelson Mozer; GOMES FILHO, Ary; SILVA, Sandro Fernandes da; FERNANDES FILHO, José. Indicadores antropométricos de obesidade em portadores da síndrome de 
http://dx.doi.org/10.5902/1984686X35273

Down entre 15 e 44 anos. Revista Brasileira de Educação Física e Esporte, São Paulo, v.23, n.4, p.415-24, out./dez. 2009.

SOUSA, Gabriel Renaldo de; PINTO, Marília Garcia; SEEBER, Jefferson Roberto; SILVA, Diego Augusto Santos. Associação do estado nutricional com aptidão física relacionada à saúde em adultos com deficiência intelectual. Revista Brasileira de Educação Física e Esporte, São Paulo; v. 29, n. 4, p. 543-50. Out-Dez, 2015.

SOUZA, Ana Cláudia Nogueira de Melo; RODRIGUES, Marina Costa; FERREIRA, Lívia Garcia. Excesso de peso e gordura corporal em portadores de síndrome de Down de uma instituição no município de Divinópolis -MG. Revista Brasileira de Ciências da Saúde, a. 11, n 37, jul/set, 2013.

TOMAZ, Rodrigo Victor Viana; ROSA, Thiago Lusivo; VAN, David Bui; MELO Débora Gusmão. Políticas públicas de saúde para deficientes intelectuais no Brasil: uma revisão integrativa. Ciências \& Saúde Coletiva, 21(1):155-172,2016.

UNICAMP. Terapia nutricional: condutas do nutricionista. Grupo de Apoio Nutricional; Equipe Multiprofissional de Terapia Nutricional; GAN / EMTN - HC; HOSPITAL DAS CLÍNICAS. Dezembro, 2003.

VERSTRAELEN, C. J. F.; MAASKANT, M. A.; KNIJFF-RAEVEN, A. G. M. van; CURFS, L. M. G.; SCHROJENSTEIN LANTMAN, H. M. J. V. Weighting the weights: agreement among anthropometric indicators identifying the weight status of people with intellectual disabilities. Journal of Applied Research in Intellectual Disabilities, 2009, 22, p. 307313.

VIEIRA, Sônia. Introdução à bioestatística. Rio de Janeiro: Campus, 1980.

VIEIRA, Sônia. Introdução à bioestatística. 3aㅗ ed. Rio de Janeiro: Campus, 1991.

WINTER, C. F. et al. Overweight and obesity in older people with intellectual disability. Elsevier, v. 33, n. 2, p. 398-405, March - April, 2012.

WOUTERS, M.; EVENHUIS, H. M.; HILGENKAMP, T. I. M. Systematic review of field based physical fitness tests for children and adolescents with intellectual disabilities.

Research in Developmental Disabilities, v. 61, p. 77- 94, 2017.

\section{Notas}

\footnotetext{
${ }^{1}$ Software de nutrição utilizado para realização de avaliação nutricional e planejamento dietético.

${ }^{2} \mathrm{O}$ presente estudo pertence a uma pesquisa maior, denominada "Estado Nutricional e Consumo Alimentar de Portadores de Necessidades Especiais", aprovada pelo Comitê de Ética em Pesquisa do Centro Universitário São Lucas, sob o nํㅜㄴ.444.940.
} 


\section{Correspondência}

Cristiane Makida Dyonisio - Centro Universitário São Lucas, Rua Alexandre Guimarães, 1927, Areal, Porto Velho, Rondônia - Brasil.

CEP: $76805-846$

\section{(c) (7) (8)}

4.0 International (CC BY-NC 4.0) 